\title{
A Non-oscillatory Advection Operator for the Compatible Spectral Element Method
}

\author{
M.A. Taylor ${ }^{1, \star}$, A. St.Cyr ${ }^{2, \star \star}$, and A. Fournier ${ }^{2}$ \\ 1 Sandia National Laboratories, Albuquerque NM 87185 \\ mataylo@sandia.gov \\ ${ }^{2}$ National Center for Atmospheric Research ${ }^{\star \star}$, Boulder CO 80303
}

\begin{abstract}
The spectral element method is well known as an efficient way to obtain high-order numerical solutions on unstructured finite element grids. However, the oscillatory nature of the method's advection operator makes it unsuitable for many applications. One popular way to address this problem is with high-order discontinuous-Galerkin methods. In this work, an alternative solution which fits within the continuous Galerkin formulation of the spectral element method is proposed. Making use of a compatible formulation of spectral elements, a natural way to implement conservative non-oscillatory reconstructions for spectral element advection is shown. The reconstructions are local to the element and thus preserve the parallel efficiency of the method. Numerical results from a low-order quasi-monotone reconstruction and a higher-order signpreserving reconstruction are presented.
\end{abstract}

\section{Introduction}

The spectral element method (SEM) with inexact numerical integration is a generalized continuous Galerkin method [1]. It is $h$ - $p$ capable, relies on globally continuous polynomial basis functions and the equations of interest are solved in integral form. The unique feature of the spectral element method is that if the elements are restricted to quadrilaterals, the integrals can be approximated by highly accurate Gauss-Lobatto quadrature rules within each element. This allows the construction of compactly supported, globally continuous basis and test functions which are orthogonal, leading to a diagonal mass matrix. The diagonal mass matrix allows time-dependent geophysical problems to be solved with simple explicit or semi-implicit methods and thus the method remains efficient while retaining the geometric flexibility of unstructured finite element grids. The method has proven accurate and effective for a wide variety of geophysical problems, including global atmospheric circulation modeling [2 34567], ocean modeling 89], and planetary-scale seismology [10]. The method has unsurpassed

\footnotetext{
* Supported in part by DOE/BER FWP06-13194.

** Supported in part by DOE DE-FG02-07ER64464 and NSF CMG-0530845.

${ }^{\star \star \star}$ NCAR is operated by the University Corporation for Atmospheric Research and sponsored by the National Science Foundation.
} 
parallel performance. It was used for earthquake modeling by the 2003 Gordon Bell Best Performance winner [11] and has successfully scaled to $\sim 100,000$ processors 1213 .

One caveat of the SEM has its source in the advection operator. For advection, the SEM can achieve excellent accuracy in the $L_{2}$ norm mainly because it uses relatively high-degree polynomials (typically between degree 4 and 10). However, the fact that the basis functions are globally continuous makes it difficult to preserve discrete analogs of other important practical properties of advection such as monotonicity and positivity. Traditional SEM results are quite oscillatory 14. In this work, it is shown how to incorporate local element reconstructions within a strong-stability-preserving (SSP) time-integrator for the compatible SEM formulation, which yield efficient non-oscillatory advection schemes.

\subsection{The Spectral Element Method}

Let $\Omega$ represent our computational domain. We first mesh $\Omega$ using a quadrilateral finite-element mesh with $M$ elements denoted $\left\{\Omega_{m}\right\}_{m=1}^{M}$. Here the focus is on the case where $\Omega$ is the surface of the sphere, and we employ a cubed-sphere based tiling of the sphere with quadrilaterals as shown in Fig. 1, It is assumed that the mesh has no hanging nodes, and that each element can be $C^{1}$ mapped to the reference element $[-1,1]^{2}$. We denote this map and its inverse by $\boldsymbol{r}=\boldsymbol{r}(\boldsymbol{x} ; m)$ and $\boldsymbol{x}=\boldsymbol{x}(\boldsymbol{r} ; m)$, where $\boldsymbol{x}=\left(x^{1}, x^{2}\right)$ are the coordinates of a point in the reference element $[-1,1]^{2}$ and $\boldsymbol{r}=\left(r^{1}, r^{2}\right) \in \Omega$. Within $[-1,1]^{2}$ we work in the space of polynomials up to degree $d$, denoted

$$
\mathcal{P}_{d}=\underset{i, j=0}{d}\left\{\phi_{i j}\right\}
$$

where $\phi_{i j}(\boldsymbol{x})=\varphi_{i}\left(x^{1}\right) \varphi_{j}\left(x^{2}\right)$ are the cardinal-functions (Lagrange interpolating polynomials) of the degree $d$ Gauss-Lobatto nodes $\xi_{i}, i=0, \ldots, d$. The cardinalfunction expansion coefficients of a function $g$ are its Gauss-Lobatto node values, so we have

$$
g(\boldsymbol{x})=\sum_{i, j=0}^{d} g\left(\xi_{i}, \xi_{j}\right) \phi_{i j}(\boldsymbol{x}) \quad \forall g \in \mathcal{P}_{d} .
$$

The SEM uses global piecewise polynomial spaces $\mathcal{H}_{d}^{0}$ and $\mathcal{H}_{d}^{1}$ defined as

$$
\begin{aligned}
& \mathcal{H}_{d}^{0}=\left\{f \in L^{2}(\Omega): f(\boldsymbol{r}(\boldsymbol{x} ; m)) \in \mathcal{P}_{d}, \forall m\right\}, \\
& \mathcal{H}_{d}^{1}=C^{0}(\Omega) \cap \mathcal{H}_{d}^{0} .
\end{aligned}
$$

Functions in $\mathcal{H}_{d}^{0}$ are polynomial in the mapped variable within each element, and $\mathcal{H}_{d}^{1}$ is the subset of these functions which are continuous across element boundaries. Let $M_{d}=\operatorname{dim} \mathcal{H}_{d}^{0}=(d+1)^{2} M$, and $L=\operatorname{dim} \mathcal{H}_{d}^{1}<M_{d}$.

For functions $f \in \mathcal{H}_{d}^{0}$, we will rely on the cardinal-function expansion local to each element

$$
f(\boldsymbol{r}(\boldsymbol{x} ; m))=\sum_{i, j=0}^{d} \hat{f}_{i j}^{m} \phi_{i j}(\boldsymbol{x}),
$$




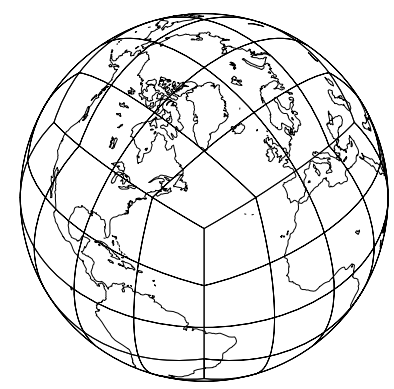

Fig. 1. Tiling the surface of the sphere with quadrilaterals. An inscribed cube is projected to the surface of the sphere. The faces of the cubed-sphere are further subdivided to form a quadrilateral grid of the desired resolution. The Gnomonic equal angle projection is used, resulting in a quasi-uniform but non-orthogonal grid [15].

where the expansion coefficients are the function values at the Gauss-Lobatto nodes, $\hat{f}_{i j}^{m}=f\left(\boldsymbol{r}\left(\xi_{i}, \xi_{j} ; m\right)\right)$. Since functions in $\mathcal{H}_{d}^{0}$ can be multi-valued at GaussLobatto points shared by more than one element, this local expansion representation will contain all such values. For $f \in \mathcal{H}_{d}^{1}$, the values at any multiply represented points must all be the same. Note that since $f(\boldsymbol{r}(\boldsymbol{x} ; m))$ is a polynomial of degree $d$ in $\boldsymbol{x}$ and there are $d+1$ Gauss-Lobatto points along each edge, then agreement at these points means we also have agreement along the entire edge, as required for $\mathcal{H}_{d}^{1}$. We note that a global piecewise cardinal function basis for $\mathcal{H}_{d}^{1}$ can be constructed by piecing together appropriate combinations of the $\phi_{i j}$ for either conforming [4] or non-conforming element meshes [16].

\subsection{The SEM Divergence Operator in Curvilinear Coordinates}

We denote the $3 \times 3$ Jacobian matrix of the mapping from $[-1,1]^{2}$ to $\Omega_{m}$ by $\mathbf{J}^{m}$, with coefficients $\left(\mathbf{J}^{m}\right)_{\alpha \beta}=\partial r^{\alpha} / \partial x^{\beta}$ and determinant $J^{m}=\left|\mathbf{J}^{m}\right|$. A vector $\boldsymbol{v}$ has contravariant components $v^{\alpha}=\boldsymbol{v} \cdot \nabla x^{\alpha}$ and covariant components $v_{\beta}=$ $\boldsymbol{v} \cdot \partial \boldsymbol{r} / \partial x^{\beta}$. The divergence operator in $\Omega_{m}$ is given by

$$
\nabla \cdot \boldsymbol{v}=\frac{1}{J^{m}} \sum_{\alpha} \frac{\partial}{\partial x^{\alpha}}\left(J^{m} v^{\alpha}\right)
$$

To compute this operator, the term $J^{m} v^{\alpha}$ that appears is first projected into $\mathcal{H}_{d}^{0}$ via interpolation at the Gauss-Lobatto grid points and then this interpolant is differentiated exactly with respect to $x^{\alpha}$ by differentiating (3). We denote the interpolation operator by $\mathcal{I}$. The sum of partial derivatives are then divided by $J^{m}$ at the Gauss-Lobatto nodal values and thus

$$
\nabla \cdot \boldsymbol{v} \approx \nabla_{h} \cdot \boldsymbol{v}=\mathcal{I}\left(\frac{1}{J} \sum_{\alpha}^{m} \frac{\partial}{\partial x^{\alpha}}\left(\mathcal{I}\left(J^{m} v^{\alpha}\right)\right)\right) \in \mathcal{H}_{d}^{0},
$$

where $\nabla_{h} \cdot()$ is the SEM divergence operator. In what follows, only the SEM operators will be employed, never the continuum operators, and thus the $h$ subscript will be dropped. 


\subsection{The SEM Inner Product}

Instead of using exact integration of the basis functions as in finite-element method, the SEM uses a Gauss-Lobatto quadrature approximation for the inner product. The following unlabeled integral is defined as the usual area weighted integral over the entire domain $\Omega$. This integral is written as a sum of integrals over the set $\left\{\Omega_{m}\right\}$ of elements used to decompose the domain:

$$
\int f g=\sum_{m=1}^{M} \int_{\Omega_{m}} f g=\left.\left.\sum_{m=1}^{M} \iint_{[-1,1]^{2}} f\right|_{\Omega_{m}} g\right|_{\Omega_{m}} J^{m} \mathrm{~d} x^{1} \mathrm{~d} x^{2} .
$$

The integral over $[-1,1]^{2}$ is approximated as

$$
\langle f, g\rangle_{\Omega_{m}}=\sum_{i, j=0}^{d} w_{i} w_{j} J^{m}\left(\xi_{i}, \xi_{j}\right) \hat{f}_{i j}^{m} \hat{g}_{i j}^{m}
$$

by using the Gauss-Lobatto quadrature points $\left\{\xi_{k}\right\}_{k=0}^{d}$ and weights $\left\{w_{k}\right\}_{k=0}^{d}$. The SEM approximation to the global integral is then naturally defined as

$$
\langle f, g\rangle=\sum_{m=1}^{M}\langle f, g\rangle_{\Omega_{m}} \simeq \int f g
$$

which is extended to vectors in the usual manner,

$$
\langle\boldsymbol{u}, \boldsymbol{v}\rangle_{\Omega_{m}}=\sum_{i, j=0}^{d} w_{i} w_{j} J^{m}\left(\xi_{i}, \xi_{j}\right) \boldsymbol{u}\left(\boldsymbol{r}\left(\xi_{i}, \xi_{j} ; m\right)\right) \cdot \boldsymbol{v}\left(\boldsymbol{r}\left(\xi_{i}, \xi_{j} ; m\right)\right) \text {. }
$$

\subsection{The Discrete Divergence Theorem within an Element}

We will also define a Gauss-Lobatto quadrature approximation to the line integral over the boundary of $\Omega_{m}$ as

$$
\langle\boldsymbol{v} \cdot \hat{n}\rangle_{\partial \Omega_{m}} \simeq \oint_{\partial \Omega_{m}} \phi \boldsymbol{v} \cdot \hat{n} d s
$$

with $d s$ being the arc length measure and $\hat{n}$ the outward unit normal. Some algebra will show that the natural Gauss-Lobatto approximation to this integral in curvilinear coordinates is

$$
\langle\boldsymbol{v} \cdot \hat{n}\rangle_{\partial \Omega_{m}}=\left.\sum_{\alpha \neq \beta} \sum_{i=0}^{d} w_{i}\left(J^{m} \boldsymbol{v} \cdot \nabla x^{\beta}\right)\right|_{x^{\alpha}=\xi_{i}, x^{\beta}=-1} ^{x^{\alpha}=\xi_{i}, x^{\beta}=1}
$$

where the inner sum is over all Gauss-Lobatto points along an element edge and the outer sum is over $(\alpha, \beta)=(1,2)$, representing the element edges where $x^{2}= \pm 1$ and $(\alpha, \beta)=(2,1)$, representing the element edges where $x^{1}= \pm 1$. Note that the corner nodes, which are shared by two edges of $\Omega_{m}$, appear twice in this sum.

The compatible SEM employs discrete analogs of several important integral properties of the divergence, gradient and curl operators [17. The key property 
we need here is the divergence theorem within an element. In the particular, the SEM discrete analog of

$$
\int_{\Omega_{m}} \nabla \cdot \boldsymbol{v}=\oint_{\partial \Omega_{m}} \boldsymbol{v} \cdot \hat{n} \mathrm{~d} s
$$

is given by

$$
\langle 1, \nabla \cdot \boldsymbol{v}\rangle_{\Omega_{m}}=\langle\boldsymbol{v} \cdot \hat{n}\rangle_{\partial \Omega_{m}}, \quad \forall \boldsymbol{v} \in \mathcal{H}_{d}^{1} .
$$

This property shows that the SEM will be locally conservative when solving equations in conservation form. In the SEM one does not need to compute the flux term, but Eq. 10 shows that the equation has a flux formulation and thus is locally conservative with respect to the element mass $\langle 1, \cdot\rangle_{\Omega_{m}}$.

\section{The SEM Locally Conservative Advection Operator}

Consider the advection operator on the surface of the sphere,

$$
\frac{\partial h}{\partial t}=-\nabla \cdot h \boldsymbol{v}
$$

with $\boldsymbol{v}$ prescribed and $\nabla \cdot \boldsymbol{v}=0$. This problem is analyzed using a forward Euler time step, and thus the results will naturally extend to higher order SSP time-stepping methods which are convex combinations of forward Euler steps. The resulting SEM discretization finds $h(t+\Delta t) \in \mathcal{H}_{d}^{1}$ such that

$$
\langle\psi, h(t+\Delta t)\rangle=\langle\psi, h(t)\rangle-\Delta t\langle\psi, \nabla \cdot h(t) \boldsymbol{v}(t)\rangle \quad \forall \psi \in \mathcal{H}_{d}^{1}
$$

The latter is equivalent to the following two-step process:

1. Advance the solution locally within each element,

$$
h^{*}=h(t)-\Delta t \nabla \cdot h(t) \boldsymbol{v}(t) .
$$

2. Let $h(t+\Delta t)$ be the projection of $h^{*}$ into $\mathcal{H}_{d}^{1}$. The projection is given by the unique $h(t+\Delta t) \in \mathcal{H}_{d}^{1}$ such that

$$
\langle\psi, h(t+\Delta t)\rangle=\left\langle\psi, h^{*}\right\rangle \quad \forall \psi \in \mathcal{H}_{d}^{1}
$$

Step one computes an $h^{*} \in \mathcal{H}_{d}^{0}$ that in general will not be globally continuous and thus not in $\mathcal{H}_{d}^{1}$. Projecting $h^{*}$ into $\mathcal{H}_{d}^{1}$ in the second step requires inverting the SEM mass matrix.

\section{A Quasi-Monotone SEM Advection Operator}

It is first shown that a low-order quasi-monotone SEM advection scheme can be obtained by introducing a reconstruction step between the two steps of the algorithm given above: 
1. Advance the solution locally within each element using Eq. 11,

2. Let $h^{* *}$ be the the result of a mass-preserving bilinear reconstruction of $h^{*}$ with slope limited so that no new extrema are created.

3. Let $h(t+\Delta t)$ be the projection of $h^{* *}$ into $\mathcal{H}_{d}^{1}$.

To show that this method is quasi-monotone, we first establish

Theorem 1. Suppose $\Delta t$ is chosen such that

$$
\Delta t\left|\langle h(t) \boldsymbol{v}(t) \cdot \hat{n}\rangle_{\partial \Omega_{m}}\right| \leq\langle 1,|h(t)|\rangle_{\Omega_{m}} \quad \forall m
$$

then step 1 (the SEM local element update) obeys a monotone-element-mean property,

$$
\min _{i, j} h\left(\boldsymbol{r}\left(\xi_{i}, \xi_{j} ; m\right), t\right) \leq\left\langle 1, h^{*}\right\rangle_{\Omega_{m}} /\langle 1,1\rangle_{\Omega_{m}} \leq \max _{i, j} h\left(\boldsymbol{r}\left(\xi_{i}, \xi_{j} ; m\right), t\right) .
$$

Proof. For $\langle 1,|h(t)|\rangle_{\Omega_{m}} \neq 0$, such a $\Delta t$ can always be chosen. Otherwise $h=0$ and the inequality is satisfied for all $\Delta t$. Note that the restriction on $\Delta t$ is a standard CFL condition, since $\langle 1,1\rangle_{\Omega_{m}} /\langle 1\rangle_{\partial \Omega_{m}}$ is proportional to element edge length.

To show that step 1 has the monotone-element-mean property, we first show that if $h(t) \geq 0$, then $\left\langle 1, h^{*}\right\rangle_{\Omega_{m}} \geq 0$. By Eq. 10 and the fact that $\langle 1,|h(t)|\rangle_{\Omega_{m}}=$ $\langle 1, h(t)\rangle_{\Omega_{m}}$, we have

$$
\begin{aligned}
\left\langle 1, h^{*}\right\rangle_{\Omega_{m}} & =\langle 1, h(t)\rangle_{\Omega_{m}}-\Delta t\langle 1, \nabla \cdot h(t) \boldsymbol{v}(t)\rangle_{\Omega_{m}} \\
& =\langle 1, h(t)\rangle_{\Omega_{m}}-\Delta t\langle h(t) \boldsymbol{v}(t) \cdot \hat{n}\rangle_{\partial \Omega_{m}} \geq 0 .
\end{aligned}
$$

Now consider

$$
\begin{aligned}
& g_{1}(t)=h(t)-\min _{i, j} h_{t}\left(\boldsymbol{r}\left(\xi_{i}, \xi_{j} ; m\right), t\right) \geq 0 \\
& g_{2}(t)=\max _{i, j} h\left(\boldsymbol{r}\left(\xi_{i}, \xi_{j} ; m\right), t\right)-h(t) \geq 0 .
\end{aligned}
$$

Applying the SEM advection step 1 to both $g_{1}(t)$ and $g_{2}(t)$, we have that $\left(1, g_{1}^{*}\right)_{\Omega_{m}} \geq 0$ and $\left(1, g_{2}^{*}\right)_{\Omega_{m}} \geq 0$, which is equivalent to the monotone-elementmean property.

Since $h^{*}$ computed in step 1 will not contain any new extrema relative to the min and max within $\Omega_{m}$, the slope limited reconstruction $h^{* *}$ computed in step 2 will obey the same property. It will be non-oscillatory, but only quasi-monotone since for high polynomial degree, if $h(t)$ is highly oscillatory within the element, it is possible that $h^{* *}$ will contain local extrema in one region of the element even though it does not contain any new extrema with respect to the element min and max values. The third and final step, applying the SEM projection operator, is a Jacobian weighted averaging of the values computed at different elements for the shared edges and corner points, and is thus monotone preserving.

This quasi-monotone scheme is far from optimal. Numerical results suggest it is only $2^{\text {nd }}$-order accurate when applied to smooth problems. It remains an open 
problem to determine if higher-order monotone reconstructions exist and to determine if an an exactly monotone reconstruction exists. Our initial results at higher-order reconstructions have focused on retaining only the sign-preserving property. We note that a conservative sign-preserving reconstruction always exists since in the worse case the reconstruction can simply set $h^{* *}$ to the element average which is conservative and always positive by Theorem 1.

\section{Numerical Results}

We now compare the three spectral element advection schemes: no reconstruction, the quasi-monotone reconstruction described above and a sign-preserving reconstruction. We have implemented these methods into the National Center for
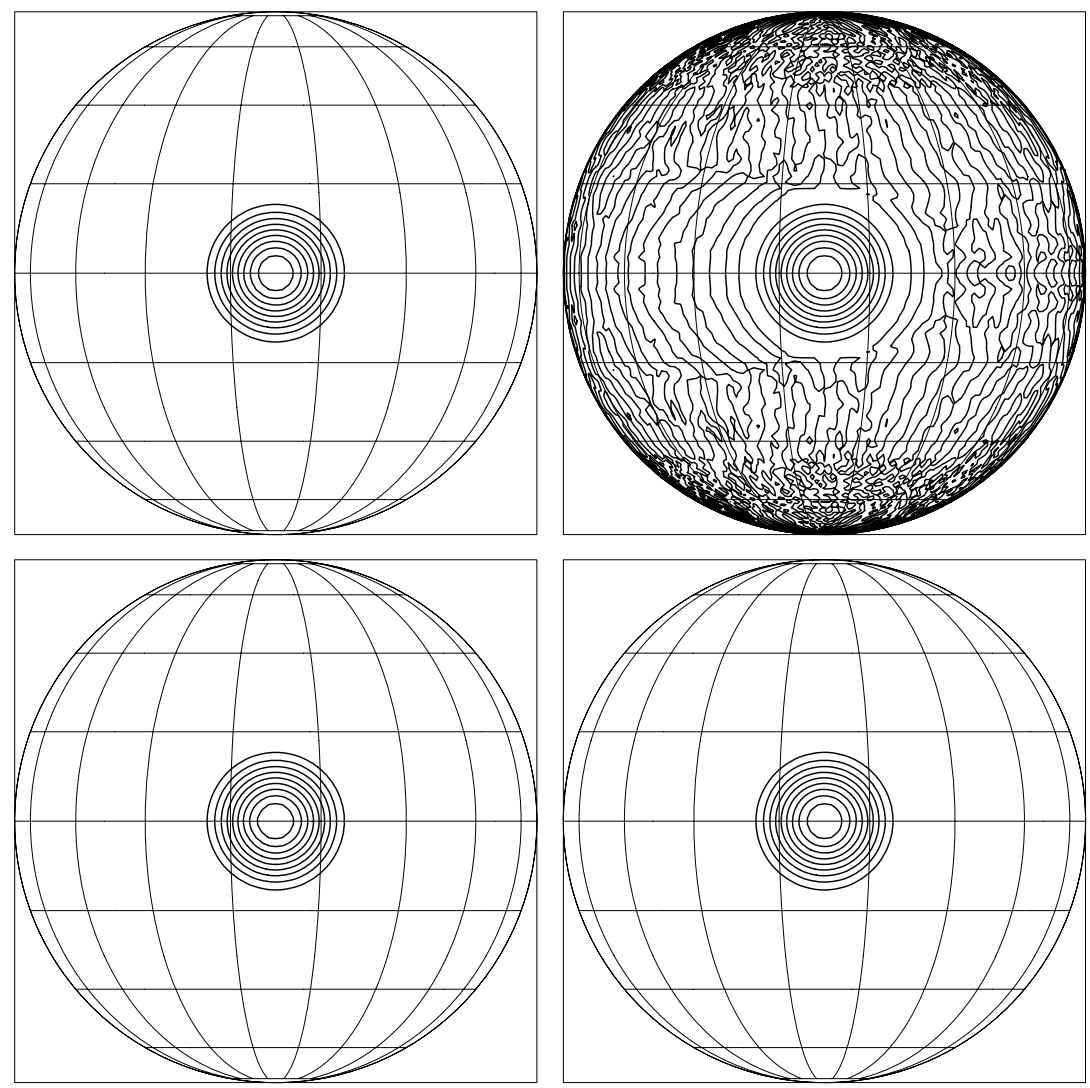

Fig. 2. Contour plots of the cosine-bell test case. Shown are the initial condition (upper left) and the solution from the un-limited advection scheme (upper right), the quasimonotone scheme (lower left) and the sign-preserving scheme (lower right). Contour lines are drawn for $h=0$ to $h=1000$ with an increment of 100 . 


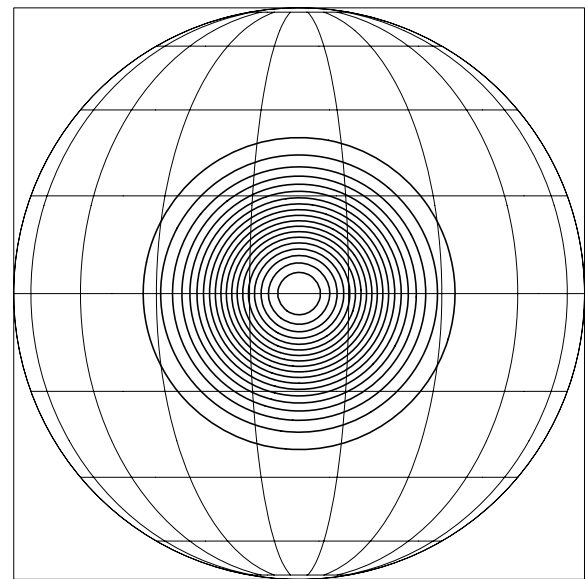

Fig. 3. Contour plot of the initial condition for the Gaussian test case. Contour lines are drawn for $h=0$ to $h=1000$ with an increment of 50 .

Atmospheric Research's High Order Method Modeling Environment (HOMME) 5. Within each face of the cubed sphere, we use an $n e \times n e$ grid of elements. Within each element we use degree $d=3$ polynomial basis functions, which is formally $4^{\text {th }}$-order accurate. The average grid spacing at the equator is $360 /(12 n e)$ degrees. We do not describe in detail our initial reconstruction algorithms since they are presented here only to demonstrate the potential of our approach.

We start with the pure advection test from the well known suite of shallowwater test cases on the sphere 18. The latter concerns the advection around the sphere of a cosine bell with compact support. The velocity is fixed (rigid rotation about the north-south axis) and the equation is integrated for 12 days or one full rotation around the sphere. Contour plots of the initial condition $h(0)$ and results from the 3 advection schemes after 12 days are shown in Fig. 2. As expected, the non-limited advection scheme is quite oscillatory, especially in the region where the solution should be zero, but has very little dissipation: the maximum of $h$ is reduced from 1000.0 to 994.0 after 12 days, while the minimum is -5.97 . Both the sign-preserving and monotone schemes completely eliminate these oscillations but have slightly more dissipation, reducing the maximum after 12 days to 992.7 and 959.0, respectively. The minimum of $h$ is zero for both of these methods.

The cosine bell has a kink at the edge of the bell where $h=0$ and thus none of the methods can achieve convergence greater than second order. To study the order of accuracy of these methods, we modify the test and instead advect a smooth Gaussian hill, shown in Fig. 3. We compute $l_{2}$ and $l_{\infty}$ errors using the same normalization as used for the first test as specified in [18. These errors are plotted in Fig. 4 for resolutions of $n e=9$ up to $n e=41$. As expected, the non-limited spectral element advection is $4^{\text {th }}$-order accurate even on the non-orthogonal unstructured cubed-sphere grid. The sign-preserving advection 

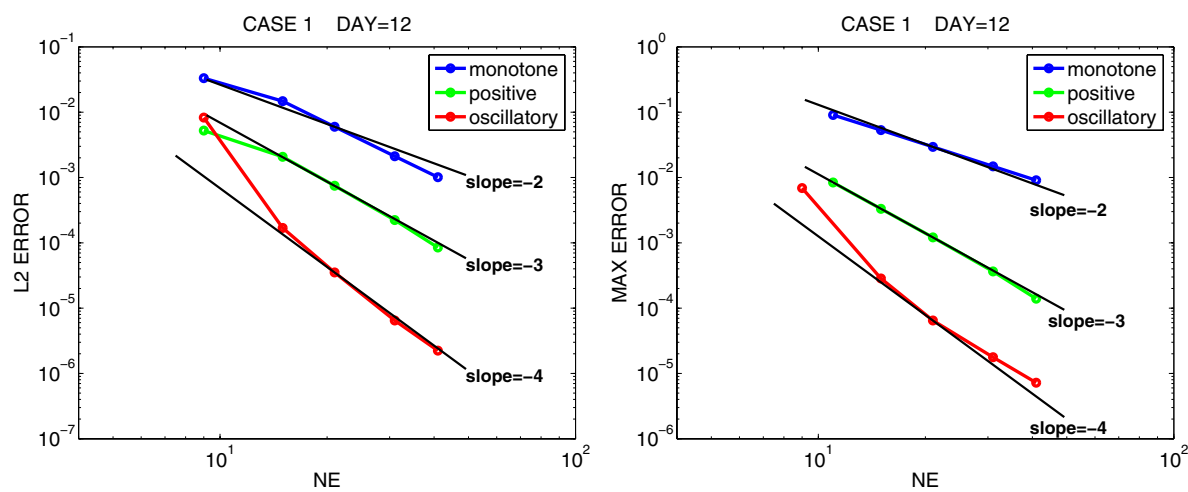

Fig. 4. Convergence of the $l_{2}$ and $l_{\infty}$ error as a function of resolution parameter ne. The non-limited spectral element scheme is labeled oscillatory, while positive refers to the scheme with a positive-preserving reconstruction and monotone is the scheme with a quasi-monotone reconstruction.

scheme loses one order of accuracy, and the monotone scheme reduces the accuracy of the scheme to second order.

\section{Conclusions}

In this work it was shown how to naturally include a function-limiting procedure within the compatible SEM formulation for the time-dependent pure advection equation. We then demonstrated that quasi-montone and sign-preserving advection schemes are obtainable by the SEM. The schemes are far from optimal and thus future work will focus on fully monotone and higher-polynomial-degree reconstruction procedures.

\section{References}

1. Maday, Y., Patera, A.T.: Spectral element methods for the incompressible Navier Stokes equations. In: Noor, A.K., Oden, J.T. (eds.) State of the Art Surveys on Computational Mechanics, pp. 71-143. ASME, New York (1987)

2. Taylor, M., Tribbia, J., Iskandarani, M.: The spectral element method for the shallow water equations on the sphere. J. Comput. Phys. 130, 92-108 (1997)

3. Giraldo, F.X.: A spectral element shallow water model on spherical geodesic grids. International Journal for Numerical Methods in Fluids 35, 869-901 (2001)

4. Fournier, A., Taylor, M., Tribbia, J.: The spectral element atmosphere model (SEAM): High-resolution parallel computation and localized resolution of regional dynamics. Mon. Wea. Rev. 132, 726-748 (2004)

5. Thomas, S., Loft, R.: The NCAR spectral element climate dynamical core: Semiimplicit eulerian formulation. J. Sci. Comput. 25, 307-322 (2005)

6. Dennis, J., Fournier, A., Spotz, W.F., St -Cyr, A., Taylor, M.A., Thomas, S.J., Tufo, H.: High resolution mesh convergence properties and parallel efficiency of a spectral element atmospheric dynamical core. Int. J. High Perf. Comput. Appl. 19, 225-235 (2005) 
7. Wang, H., Tribbia, J.J., Baer, F., Fournier, A., Taylor, M.A.: A spectral element version of CAM2. Monthly Weather Review 135 (2007)

8. Haidvogel, D., Curchitser, E.N., Iskandarani, M., Hughes, R., Taylor, M.A.: Global modeling of the ocean and atmosphere using the spectral element method. Atmosphere-Ocean Special 35, 505-531 (1997)

9. Molcard, A., Pinardi, N., Iskandarani, M., Haidvogel, D.: Wind driven circulation of the mediterranean sea simulated with a spectral element ocean model. Dynamics of Atmospheres and Oceans 35, 97-130 (2002)

10. Komatitsch, D., Tromp, J.: Spectral-element simulations of global seismic wave propagation - I. validation. Geophys. J. Int. 149, 390-412 (2002)

11. Komatitsch, D., Tsuboi, S., Ji, C., Tromp, J.: A 14.6 billion degrees of freedom, 5 teraflops, 2.5 terabyte earthquake simulation on the earth simulator. In: Proceedings of the ACM / IEEE Supercomputing SC 2003 conference (2003)

12. Bhanot, G., Dennis, J.M., Edwards, J., Grabowski, W., Gupta, M., Jordan, K., Loft, R.D., Sexton, J., St-Cyr, A., Thomas, S.J., Tufo, H.M., Voran, T., Walkup, R., Wyszogrodski, A.A.: Early experiences with the 360TF IBM BlueGene/L platform. International Journal of Computational Methods 5, 237-253 (2008)

13. Taylor, M.A., Edwards, J., St-Cyr, A.: Petascale atmospheric models for the community climate system model: New developments and evaluation of scalable dynamical cores. J. Phys. Conf. Ser. 125(012023) (2008)

14. Iskandarani, M., Levin, J., Choi, B.J., Haidvogel, D.: Comparison of advection schemes for high-order $\mathrm{h} p$ finite element and finite volume methods. Ocean Modelling 10, 233-252 (2005)

15. Rančić, M., Purser, R., Mesinger, F.: A global shallow-water model using an expanded spherical cube: Gnomonic versus conformal coordinates. Q. J. R. Meteorol. Soc. 122, 959-982 (1996)

16. Fournier, A., Rosenberg, D., Pouquet, A.: Dynamically adaptive spectral-element simulations of $2 \mathrm{~d}$ incompressible navier-stokes vortex decays. Geophysical and Astrophysical Fluid Dynamics (2009) (to appear)

17. Taylor, M.A., Edwards, J., Thomas, S., Nair, R.: A mass and energy conserving spectral element atmospheric dynamical core on the cubed-sphere grid. J. Phys. Conf. Ser. 78(012074) (2007)

18. Williamson, D.L., Drake, J.B., Hack, J.J., Jakob, R., Swarztrauber, P.N.: A standard test set for numerical approximations to the shallow water equations in spherical geometry. J. Comput. Phys. 102, 211-224 (1992) 